\title{
Laser mode complexity analysis in infrared waveguide free-electron lasers
}

\author{
Rui Prazeres* \\ Laboratoire de Chimie Physique CNRS, Université Paris-Sud, Université Paris-Saclay Bâtiment 201 P2, \\ 91405 Orsay, France
}

(Received 26 January 2016; published 20 June 2016)

\begin{abstract}
We analyze an optical phenomenon taking place in waveguide free-electron lasers, which disturbs, or forbids, operation in far infrared range. Waveguides in the optical cavity are used in far-infrared and $\mathrm{THz}$ ranges in order to avoid diffraction optical losses, and a hole coupling on output mirror is used for laser extraction. We show that, when the length of the waveguide exceeds a given limit, a phenomenon of "mode disorder" appears in the cavity, which makes the laser difficult, or impossible, to work properly. This phenomenon is even more important when the waveguide covers the whole length of the cavity. A numerical simulation describes this effect, which creates discontinuities of the laser power in the spectral domain. We show an example with an existing infrared Free-Electron Laser, which exhibits such discontinuities of the power, and where no convincing explanation was proposed until now.
\end{abstract}

DOI: 10.1103/PhysRevAccelBeams.19.060703

\section{INTRODUCTION}

Several infrared free-electron lasers (FEL) are presently used, around the world, as light sources by a large scientific community $[1,2]$. FELs are especially efficient in infrared, producing high power and large wavelength tunability over typically 1 to 2 decades. However, at large wavelength, the optical mode transverse size tends to undergo losses at the undulator vacuum chamber. The size of this chamber cannot be increased if one wants to maintain a sufficiently strong undulator magnetic field on-axis. Therefore, several infrared FELs [3-6] have designed the vacuum chamber so as to use it as an optical waveguide. Some of them are using a full waveguide cavity [6]: the waveguide covers the whole length between the cavity mirrors. The other FELs are using a partial guided cavity [3-5]: a combination of waveguide, in the undulator section, and of "free space" areas close to the cavity mirrors. The laser power extraction is generally performed by a hole coupling on the output mirror of the cavity, since no wideband mirror exists in infrared and most parts of substrates contain absorption bands.

In this last configuration of partial guided cavity, a phenomenon of "spectral gaps" has already been observed [7]: the laser power falls down at some particular wavelengths in the tuning range, whatever the beam adjustments are. We have shown that this effect is related to a combination of two effects: (1) cavity losses at the

\footnotetext{
rui.prazeres@u-psud.fr

Published by the American Physical Society under the terms of the Creative Commons Attribution 3.0 License. Further distribution of this work must maintain attribution to the author $(s)$ and the published article's title, journal citation, and DOI.
}

entrances of the waveguide (optical losses when the laser pulse goes from free space into the waveguide), and (2) bad output coupling when the laser profile exhibits a minimum of amplitude upon the extraction hole. The relevant wavelength of the "spectral gaps" depends on the waveguide dimensions. As an example, the CLIO FEL exhibits one power drop within his tuning range, close to $\lambda=60 \mu \mathrm{m}$ (see in Sec. 3). It is not too annoying if the parameters of the waveguide are well chosen to reduced the number of "power gaps" in the range: CLIO works as a reliable user facility and is not much adversely affected by this phenomenon.

In the other configuration, i.e., for full cavity waveguide, the first effect (1) should not be observed, since the waveguide goes from one cavity mirror to the next, and the extremities of the waveguide are no longer generating losses. The only source of cavity losses is the hole coupling extraction mirror (except bad reflectivity of the cavity mirrors or waveguide walls). Nevertheless, a similar, but stronger, phenomenon of power drop has been measured on FLARE [6], the infrared FEL installed in Radboud University (Nederland), which uses a full waveguide configuration. Indeed, as shown in Fig. 1, the wavelength dependence of FEL output shows a large number of power drops, which seems to be randomly distributed, and create discontinuities in the laser spectral domain. Note that the spiky spectrum is reproducible in time, from one day to the next, and it does not correspond to a chaotic dynamic which would give an unpredictable behavior. This discontinuity is penalizing for the laser users, and no convincing explanation was proposed until now. We give here an answer, based on numerical simulations, which allows us to reproduce such discontinuities in the spectral domain. We will also explain which are the limit conditions leading to such behavior. 


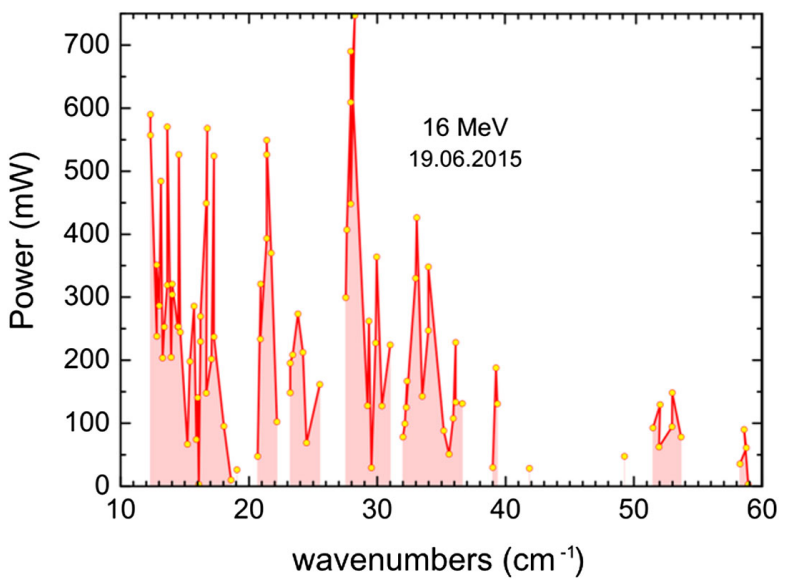

FIG. 1. Output laser power vs. wavelength, measured on FLARE at $16 \mathrm{MeV}$.

\section{NUMERICAL SIMULATION OF FLARE}

A numerical simulation has been used here, with the code MODES [8-10], already used previously to describe the "spectral gaps" phenomenon [7]. This code calculates the transverse profile $A(x, y)$ of the laser, sampled by an array of $256 \times 256$ elements. It uses an iterative process, starting from a Gaussian distribution array $A_{o}(x, y)$, and calculating the propagation of the laser wave in the optical cavity, for a certain number $N_{c}$ of cavity round trips, until convergence toward the saturation mode. The number of round trips may be from about $N_{c}=10$ up to 500. Figure 2 shows a typical example of convergence of the cavity losses, using a criterion of stability $<0.5 \%$.

The profile $A(x, y)$ is calculated at various places inside the optical cavity. The code uses the Fast Fourier Transform (FFT) to calculate the wave propagation in free space. The calculation of propagation in the waveguide section uses an operator of symmetry, called Mosaic transform [9], which is applied before the FFT operator. This Mosaic operator reproduces the transverse symmetry of the waveguide. Two different Mosaic operators are existing, with different

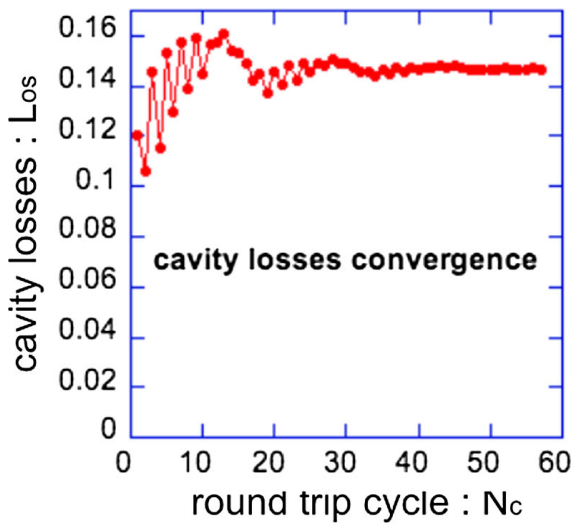

FIG. 2. Typical convergence of cavity losses vs cavity round trip $N_{c}$.

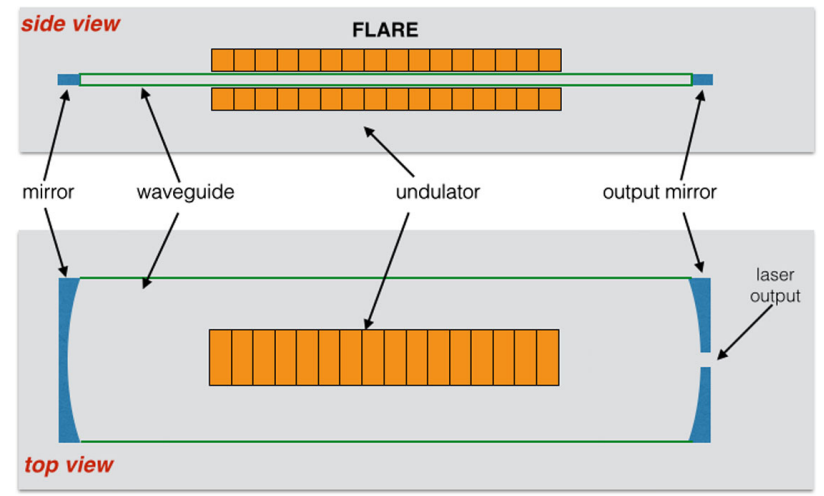

FIG. 3. Layout of FLARE Free-Electron Laser.

symmetries according to the horizontal or vertical polarization of the wave. We use here the horizontal one. The FEL gain is achieved by a product with a Gaussian transverse distribution $G(x, y)$, in the middle of the undulator section. The transverse size of $G(x, y)$ corresponds to the electron bunch transverse RMS dimensions. The code gives also the optical cavity losses, the extraction rate of the laser through the hole coupling, and the output power. The calculation of the power at laser saturation is based on the theory of FEL intensity saturation which has been developed by Dattoli et al. [11]. Note that this code uses a monochromatic wavelength, and it does not take into account the spectral nor longitudinal aspects of the laser. As a consequence, the cavity length tuning of the FEL is implicitly considered in these simulations as optimum value, at any wavelength of the laser.

In a first set of simulations, the parameters of the numerical simulation have been chosen from the FLARE FEL. The waveguide goes from one cavity mirror to the next. The transverse dimensions of the waveguide are: $a=20 \mathrm{~cm}$ in horizontal, and $b=1 \mathrm{~cm}$ in vertical. Therefore, in principle, only the vertical axis is guided. The cavity length is $d=7.5 \mathrm{~m}$. Both mirrors are of concave cylindrical type [12], and the out-coupling of the laser is achieved by a slit of $2.5 \mathrm{~mm}$ width per $1 \mathrm{~cm}$ height in the output mirror. The layout of the optical cavity is shown in Fig. 3. The main parameters of the simulation are shown in Table I.

We have taken into account here the fact that the FEL gain process is modified when a waveguide is used in the optical cavity [13]. Indeed, the laser wave may be decomposed into eigenmodes $\mathrm{TEM}_{p q}$, which the propagation speed $v_{g}(p, q)$ in the waveguide depends on numbers $(p, q)$. Therefore, for each eigenmode $\mathrm{TEM}_{p q}$ corresponds a shifted FEL resonance frequency $\lambda_{p q}$ and a shifted centroid of the gain bandwidth. This implies that some high order eigenmodes are not contributing to the FEL gain, because the laser wavelength $\lambda_{L}$ may be out of the relevant gain bandwidth. In order to take into account this effect in the numerical code, the "FEL gain operator" is 
TABLE I. Parameters used for FLARE simulations.

Optical cavity length: $7.5 \mathrm{~m}$

Mirrors radius of curvature: $4.81 \mathrm{~m}$ in horizontal

Extraction hole: $2.5 \times 10 \mathrm{~mm}$ (vertical slit)

Waveguide transverse size: $200 \times 10 \mathrm{~mm}$

Undulator period: $110 \mathrm{~mm}$

Number of undulator periods: 40

Undulator parameter Krms: $0.7-3.4$

Electron beam energy: $10-15 \mathrm{MeV}$

Energy spread: $0.8 \%$ (nominal $=0.3 \%$ )

Electron beam transverse size: $\sigma_{X Y}=5 \times 4 \mathrm{~mm}$ RMS

Bunch charge: $150 \mathrm{pC}$

Micro-pulse duration: 10 ps (nominal $=3$ ps)

Micro-pulse frequency: $3 \mathrm{GHz}$

Macro-pulse duration: $10 \mu \mathrm{s}$

Macro-pulse repetition rate: $10 \mathrm{~Hz}$

applied independently to each eigenmode. More precisely, the laser transverse profile $A(x, y)$ is decomposed into a sum of modes TEM $_{p q}$. The FEL gain is applied independently upon each individual amplitude distribution $A_{p q}(x, y)$ of the mode TEM $p q$. These amplitudes are then summed at the end of the process. The value of this gain is dependent on the mode $(p, q)$ : maximum when the laser wavelength $\lambda_{L}$ is on center of the gain bandwidth of the mode, or zero when outside. The transverse distribution $G(x, y)$ of the gain is Gaussian with rms width equal to the electron beam size in the undulator. Note that the gain $G(x, y)$ has a maximum on center, which makes a distortion of the amplitude distribution of each pure mode $A_{p q}(x, y)$, which must itself be decomposed into higher modes $\mathrm{TEM}_{p q}$. This effect creates a blending of the modes.

Nevertheless, these effects of the waveguide upon the total gain are not extremely significant, because (1) there is a blend of modes in the FEL gain process. A first reason of such blending, explained above, is due to the distortion of

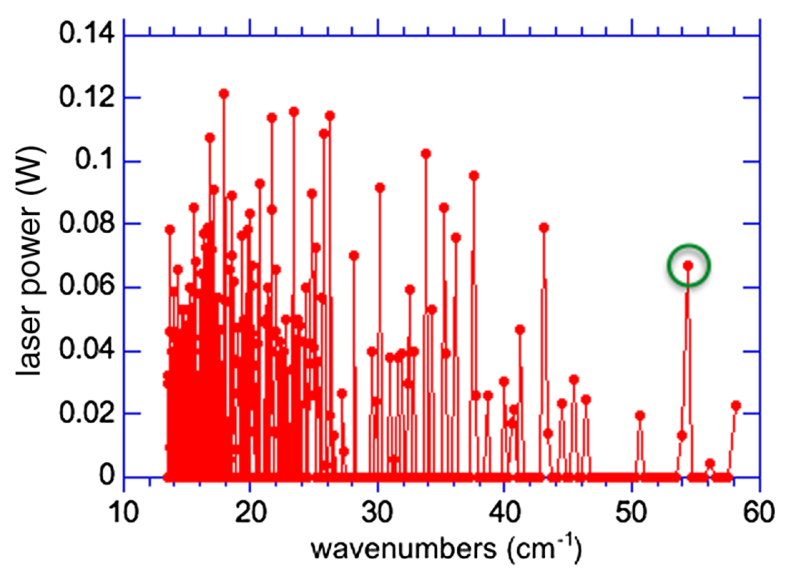

FIG. 4. Numerical simulation of the output laser power of FLARE.

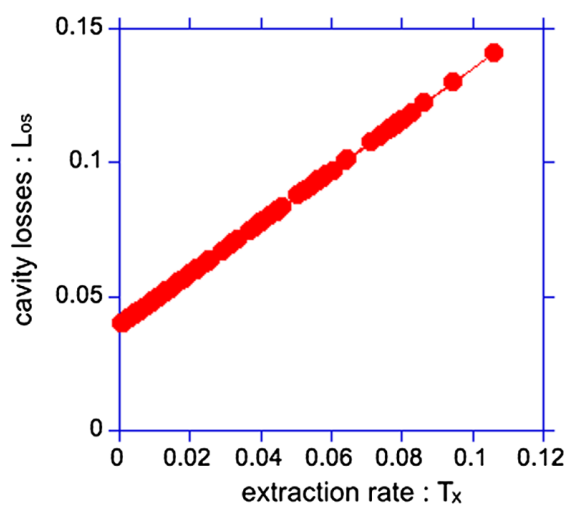

FIG. 5. Total losses $L_{\mathrm{os}}$ of the cavity versus the extraction rate $T_{x}$.

the pure modes by the gain transverse distribution. A second reason is due to diffraction on the output mirror, because of the diffraction by the hole coupling. Also (2) the laser energy on the "off gain modes" is present in the optical cavity: it does not contribute to the FEL saturation process, but it is still contributes to the extracted laser power. This is equivalent to a "Filling Factor" in the cavity. Therefore, the consequences of waveguide effect upon the gain are not so crucial for the extracted laser power.

The results of the numerical simulation, using the parameters of FLARE for $16 \mathrm{MeV}$ electron energy, are displayed in Fig. 4, and they must be compared to the experimental measurements in Fig. 1. The output laser power $P_{L}$ exhibits a discontinuity, as in measurements. Note that some parameters have been adjusted in order to fit the average value of output power: the electron energy spread is $0.8 \%$ and the electron pulse duration is $10 \mathrm{ps}$. This adjustment is necessary to compensate the approximations which are intrinsic to a numerical code, especially when it involves many parameters like for FELs, but it does not change the general behavior of the results. This curve shows that the numerical code MODES is able to simulate such a spiky behavior. The next part will show which parameters are important and are responsible of this phenomenon.

The code also gives the total cavity losses $L_{\mathrm{os}}=$ $\Delta P_{\text {in }} / P_{\text {in }}$ and the extraction rate $T_{x}=P_{\text {out }} / P_{\text {in }}$, where $P_{\text {out }}$ and $P_{\text {in }}$ are respectively the output power and inner power of the laser, and $\Delta P_{\text {in }}$ is the power loss per cavity round trip of the laser pulse. The distribution of losses $L_{\mathrm{os}}$ according to wavelength is not displayed here but it exhibits the same kind of discontinuity as in Fig. 4. However, Fig. 5 displays the distribution of total losses $L_{\mathrm{os}}$ as a function of the extraction rate $T_{x}$, calculated for each wavelength. The y-intercept of the straight line, at $T_{x}=0$, shows $L_{\mathrm{os}}=4 \%$ corresponding to reflectivity losses on both mirrors (intensity reflectivity of each mirrors $=2 \%$ ). Also, the linear distribution of points allows one to verify that no other source of loss exists in this configuration: $L_{\mathrm{os}}=0.04+T_{x}$. 


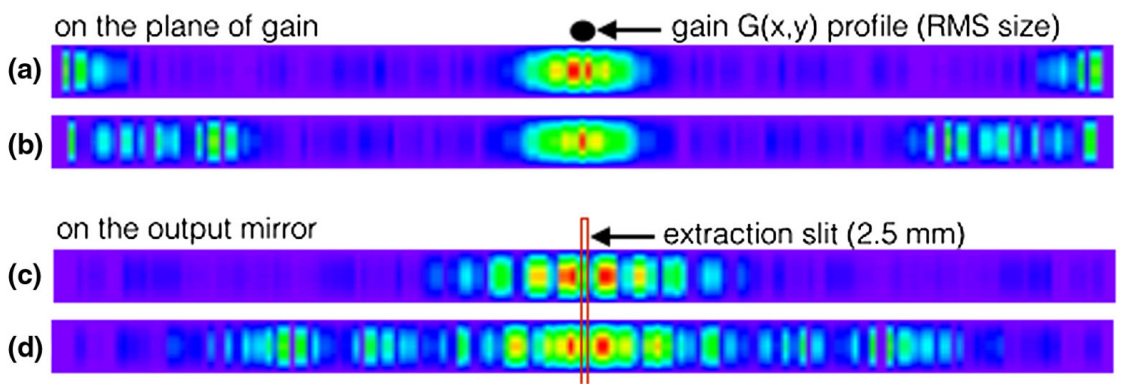

FIG. 6. Laser profile amplitude in the waveguide $(200 \times 1 \mathrm{~cm})$, at various wavelengths: large power at $54.3 \mathrm{~cm}^{-1}$ (a) $(\mathrm{c})$, and zero power at $53.5 \mathrm{~cm}^{-1}$ (b) (d); on the plane of gain (a) (b) and on the output mirror (c) (d).

Several causes may be involved in such spiky behavior: the gain, the cavity losses and the extraction rate. These parameters are strongly dependent on the transverse laser profile inside the optical cavity. Figure 6 shows a series of transverse profiles, for two wavelengths and on two different points of the cavity. The top images (a) (b) are calculated in the middle of the waveguide, in the plane of gain, whereas the images (c) (d) are calculated on the output mirror. The RMS transverse size of gain distribution $\sigma_{X Y}=5 \times 4 \mathrm{~mm}$ is represented to scale by a black dot. The images (a) (c) are for $\lambda=54.3 \mathrm{~cm}^{-1}$ corresponding to a high power point on the curve of Fig. 4 (shown with a circle), and (b) (d) are for $\lambda=53.5 \mathrm{~cm}^{-1}$ corresponding to zero output power. These profiles exhibit a complex structure in horizontal axis, which seems to vary quite randomly according to the laser wavelength. As explained in Sec. V, this structure is due to the extraction slit which makes a diffraction of the wave inside the cavity.

First, the extracted power $P_{\text {out }}$ is dependent on the extraction rate $T_{x}$, i.e., on the transverse distribution of the laser mode on the output coupling slit on the mirror, as displayed in Figs. 6(c) and 6(d). As a consequence, and as shown on left part of Fig. 7, the extraction rate $T_{x}$ also varies randomly with the wavelength. Therefore, the total losses $L_{\mathrm{os}}$ are following the variations of the extraction rate
$T_{x}$, and also exhibit spiky variations. The inner power $P_{\text {in }}$ and output power $P_{\text {out }}$ are dependent on $L_{\mathrm{os}}$, and therefore are also showing a spiky behavior. Note that laser output has a complex dependence on the extraction rate $T_{x}$ and losses $L_{\mathrm{os}}$ : large values for $T_{x}$ do not always correspond to high power, since the relevant value of losses may have a negative influence on the power. Section V describes a simulation without extraction slit. Second, the required gain coefficient also depends on the distribution of laser modeFigs. 6(a) and 6(b), because the FEL gain only occurs in a small area $\sigma_{X Y}$ on axis: the required gain coefficient $g_{o}$ must be larger when the laser profile exceed the limits of $\sigma_{X Y}$, in order to compensate the total losses $L_{\mathrm{os}}$. Note that various kinds of "gain" $[10,11]$ are used in this discussion: (1) the "required gain coefficient" $g_{o}$, which corresponds to the required value for the amplification of the laser wave $A(x, y) \cdot \operatorname{sqrt}\left[1+g_{o} . G(x, y)\right] ;$ (2) the integrated total gain of energy $G_{\text {tot }}$, with $\left(1-L_{\mathrm{os}}\right)\left(1+G_{\mathrm{tot}}\right)=1$; and (3) the FEL gain coefficient $G^{*}$ created by the electron beam, more precisely it corresponds to the "small signal gain" of laser intensity in high gain regime [11]. The laser operation only occurs when the FEL gain coefficient $G^{*}$ is large enough to exceed the required value of gain coefficient $g_{o}$. The right part of Fig. 7 displays the gain $g_{o}$ and $G^{*}$ versus wavelength. The FEL gain $G^{*}$ is intrinsic to the FEL process: it is
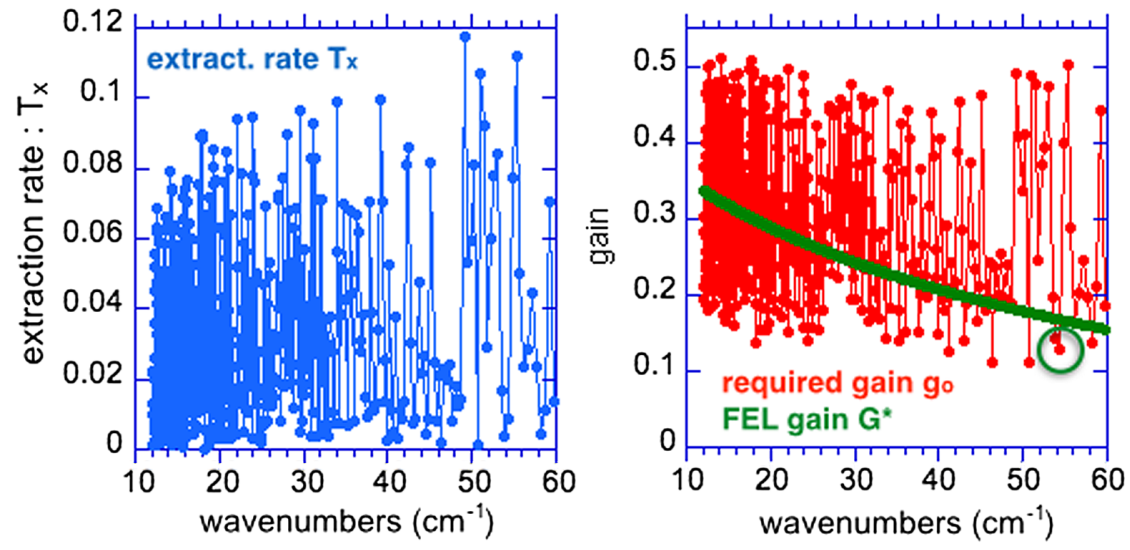

FIG. 7. Extraction rate $T_{x}$ (left side), and gain (right side) versus wavelength-required gain coefficient $g_{o}$ and FEL gain coefficient $G^{*}$. 


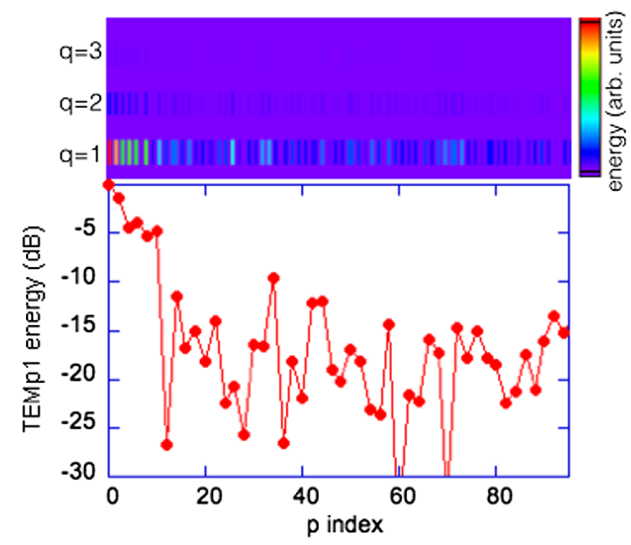

FIG. 8. Energy distribution of the eigenmodes $\mathrm{TEM}_{p q}$ of FLARE, at $\sigma=50 \mathrm{~cm}^{-1}$, versus $(p, q)$ indexes, and bottom curve in $\mathrm{dB}$ for $q=1$.

softly dependent on wavelength and has smooth variations. The required gain coefficient $g_{o}$ is strongly dependent on the laser profile, and exhibits spiky variations. The laser only occurs when $G^{*}>g_{o}$. These two effects, laser extraction and required gain variations, are responsible of the spiky behavior of the laser power observed in simulations on Fig. 4, and in measurements on Fig. 1.

\section{A. Eigenmode distribution}

The simulation code MODES does not use a decomposition in eigenmodes to calculate the wave propagation in the waveguide section. It calculates directly the propagation of the amplitude $A(x, y)$ through the waveguide. This is an important feature of the code because it takes into account the whole distribution of the laser mode, without losing the higher eigenmodes. Nevertheless, more precisely, the eigenmode decomposition is not explicitly used in the simulation code, but it is used implicitly, with a large number of modes: up to 128 modes for both $\mathrm{p}$ and $\mathrm{q}$ indexes representing an ensemble of 16384 eigenmodes. This number is very large as compared to a usual method of calculation with a decomposition into eigenmodes. In fact, in the waveguide section, the FFT, applied to the Mosaic transform of $A(x, y)$, gives directly [9] the amplitude distribution of the eigenmodes TEM TEq $_{p q}$.

An example is shown in Fig. 8, which displays the amplitude distribution of the eigenmodes $\operatorname{TEM}_{p q}$ as a function of index $(p, q)$, in the case of FLARE at $\sigma=50 \mathrm{~cm}^{-1}$. A cut is also displayed in $\mathrm{dB}$ versus $\mathrm{p}$ index for $q=1$. In this particular case, the flattened shape of the waveguide aperture $(20 \times 1 \mathrm{~cm})$ requires a large number of modes to "build" the laser profile in horizontal, because its size is small as compared to the waveguide width; whereas a few number of modes are sufficient to build it in vertical. From the example displayed in Fig. 8, we calculate that $95 \%$ of the energy is concentrated in the modes with $p \leq 95$ and $q=1$. Note that this result corresponds to the laser profile which is obtained after the convergence of the numerical process, i.e., at saturation regime. In any case, this shows that the complexity of the eigenmodes pattern does not allow to build a rather simple analytical solution of the problem.

\section{NUMERICAL SIMULATION OF A PARTIAL WAVEGUIDE WITH THE CLIO CONFIGURATION}

In order to understand which are the main FEL parameters that are responsible for the spiky behavior, it is more convenient to start from an operational configuration, and to modify the parameters, in the simulation, up to reach the spiky mode regime. Also, the FLARE configuration is not so simple to analyze since it uses cylindrical mirrors, which make a mixing between free space and waveguide respectively in horizontal and vertical. Its study will be detailed in Sec. V. Therefore, we have done the simulations starting from the configuration of the CLIO FEL, which does not show spiky features and gives a smoothed power output vs wavelength. It is easy to make this study using a numerical simulation, but quite impossible to do it experimentally because the modifications of the FEL configuration would require many months of design and developments.

Figure 9 shows the layout of the optical cavity of CLIO, and the main parameters are displayed in Table II. The waveguide length of $2 \mathrm{~m}$ only fits the undulator section, and the rest of the cavity is in free space. The laser output coupling is achieved by a hole of $2 \mathrm{~mm}$ diameter in the center of the downstream mirror. The transverse size of the gain distribution is $800 \times 400 \mu \mathrm{m}$ RMS, located in the middle of the undulator section.

The numerical simulation of the output laser power is shown in Fig. 10 as a function of wavelength, for various electron beam energy between $45 \mathrm{MeV}$ and $15 \mathrm{MeV}$. It does not exhibit a spiky behavior. A hole is observed at $\lambda=60 \mu \mathrm{m}$. It is a well-known phenomenon called

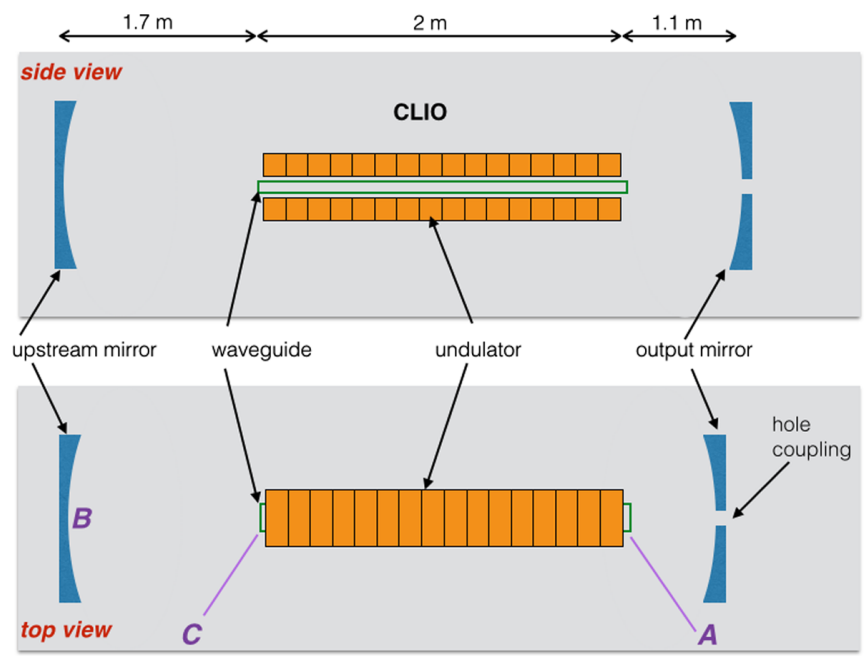

FIG. 9. Layout of CLIO Free-Electron Laser. 
TABLE II. Main parameters used for CLIO.

Optical cavity length: $4.8 \mathrm{~m}$

Waveguide length: $d_{w}=2 \mathrm{~m}$

Waveguide transverse size: $35 \times 16 \mathrm{~mm}$

Mirrors radius of curvature: $R_{c}=3 \mathrm{~m}$ (downstream), and $2.5 \mathrm{~m}$ (upstream)

Mirror reflectivity: $R_{M}=0.98$ (for intensity)

Extraction hole: $2 \mathrm{~mm}$

Undulator period: $50 \mathrm{~mm}$

Number of undulator periods: 38

Undulator parameter $K_{\mathrm{RMS}}: 0.5-2.2$

Electron beam energy: $\gamma \mathrm{mc}^{2}=15-45 \mathrm{MeV}$

Energy spread: $\sigma_{\gamma} / \gamma=0.2 \%$

Electron beam transverse size: $\sigma_{X Y}=800 \times 400 \mu \mathrm{m}$ RMS

Bunch charge: $2 \mathrm{pC}$

Micro-pulse duration: $\sigma_{t}=4 \mathrm{ps}$

Micro-pulse frequency: $31.25 \mathrm{MHz}$

Macro-pulse duration: $10 \mu \mathrm{s}$

Macro-pulse repetition rate: $25 \mathrm{~Hz}$

"spectral gap" [7], described in Sec. I, which is due to cavity losses and bad hole coupling at particular wavelengths.

In order to allow a comparison, the measurement are displayed in Fig. 11. The "spectral gap" forbids laser operation close to $\lambda=60 \mu \mathrm{m}$.

\section{A. Influence of the waveguide length}

In order to point out the influence of the waveguide length $d_{w}$, a simulation has been done using the main parameters of CLIO, with various length $d_{w}$ between 2 and $4 \mathrm{~m}$. The free spaces areas have been kept constant, therefore the total length of the cavity varies between $4.8 \mathrm{~m}$ (the real length) and $6.8 \mathrm{~m}$. Figure 12 displays the result for $\lambda=10 \mu \mathrm{m}$. The power (red curve) drops to zero when the waveguide length is larger than $2.6 \mathrm{~m}$. It is due to the decreasing of the hole coupling extraction rate $T_{x}$ and increasing of the optical losses $L_{\mathrm{os}}$. These losses are due to

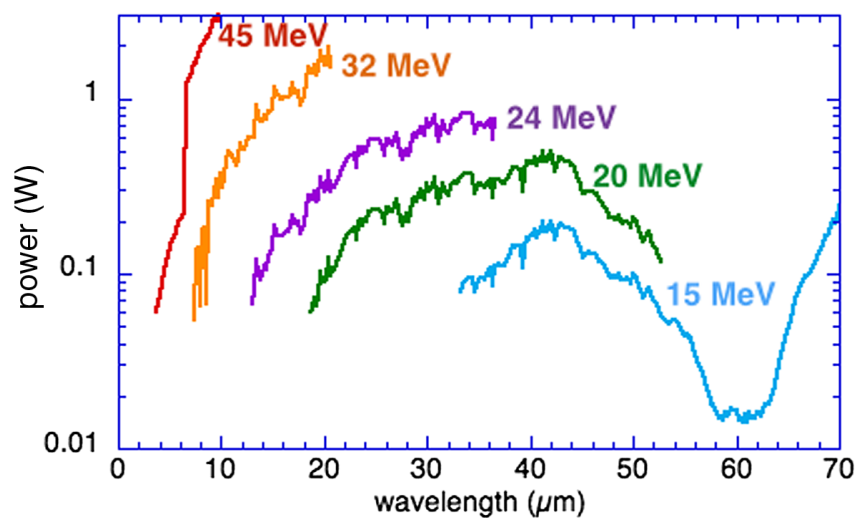

FIG. 10. Numerical simulation of the laser power vs wavelength for CLIO, for various electron beam energies.

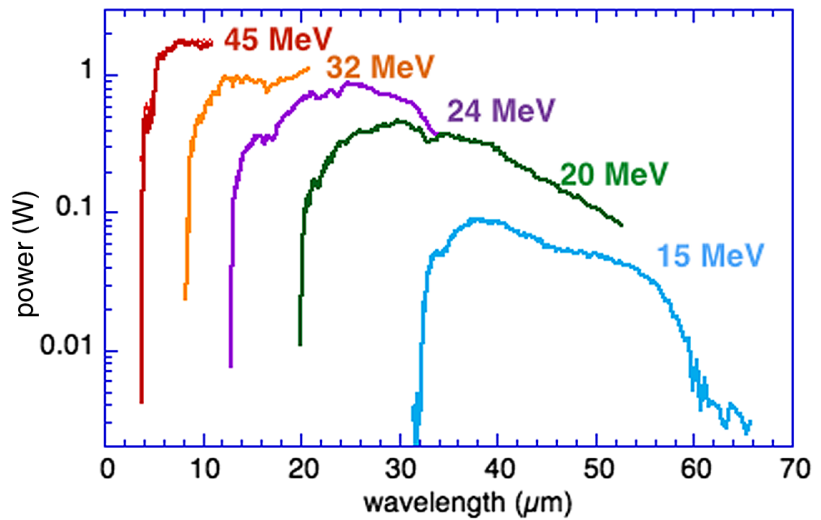

FIG. 11. Measurements of the CLIO laser power vs wavelength, for various electron beam energies.

a combination of various causes: hole extraction, mirror reflectivity and optical diffraction (the losses in the waveguide walls are neglected here). The losses $T_{x}$ due to the hole coupling are displayed in Fig. 12. The losses due to mirror reflectivity $R_{M}=0.98$ are rather small: $\left(1-R_{M}\right)=0.02$ per mirror, and are constant. The diffraction losses $L_{\text {dif }}$ correspond approximately to the difference: $L_{\text {dif }} \cong L_{\mathrm{os}}-T_{x}-2\left(1-R_{M}\right)$. Figure 13 represents the diffraction losses $L_{\text {dif }}$ as a function of waveguide length $d_{w}$, and for different wavelengths. The large contribution of diffraction is clearly visible here: the diffraction losses are increasing strongly after a threshold value for the waveguide length $d_{w}$. This threshold is close to $d_{w}=2.7 \mathrm{~m}$ and seems not depend on the wavelength.

The variations of laser power versus wavelength is shown in Fig. 14, for various waveguide length $d_{w}$. The diffraction losses $L_{\text {dif }}$ are displayed in Fig. 15. For $d_{w}=2.4 \mathrm{~m}$, just before the threshold, the power varies softly with the wavelength, and diffraction losses are small. However, for $d_{w}=3 \mathrm{~m}$, behind threshold, the power is decreased by more than 2 orders of magnitude and tends to become spiky, and the losses are larger than $20 \%$.

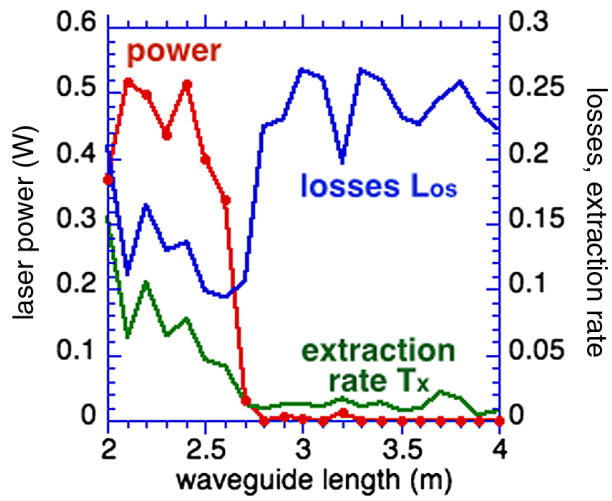

FIG. 12. Laser power $P_{L}$, cavity losses $L_{\mathrm{os}}$ and extraction rate $T_{x}$ versus waveguide length $d_{w}$, at $\lambda=10 \mu \mathrm{m}$. 


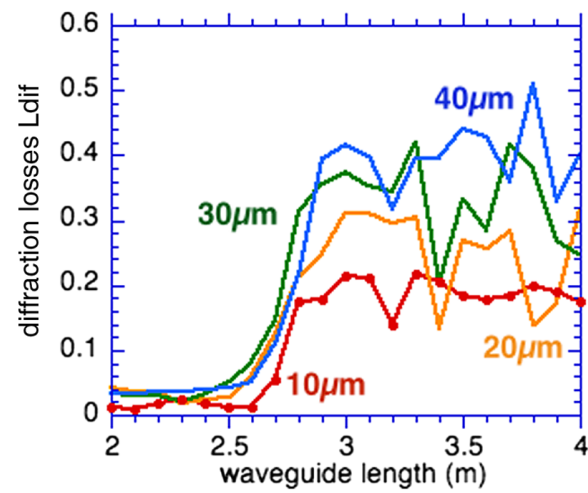

FIG. 13. Diffraction losses $L_{\text {dif }}$ versus waveguide length $d_{w}$ at various wavelength of the laser.

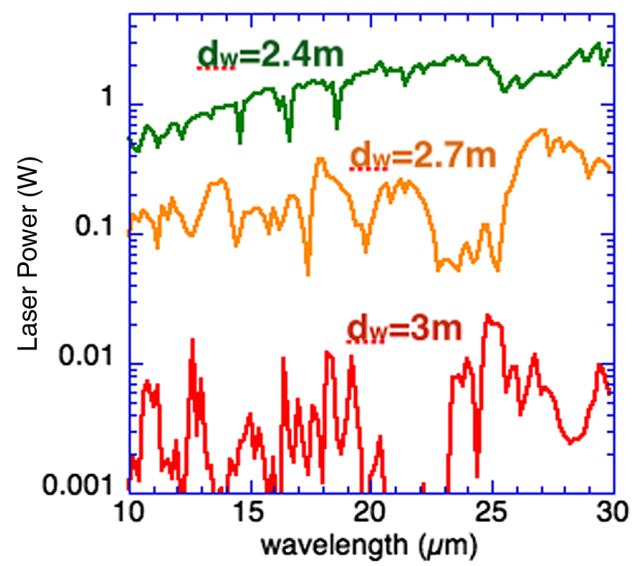

FIG. 14. Laser power versus wavelength at various waveguide length $d_{w}$.

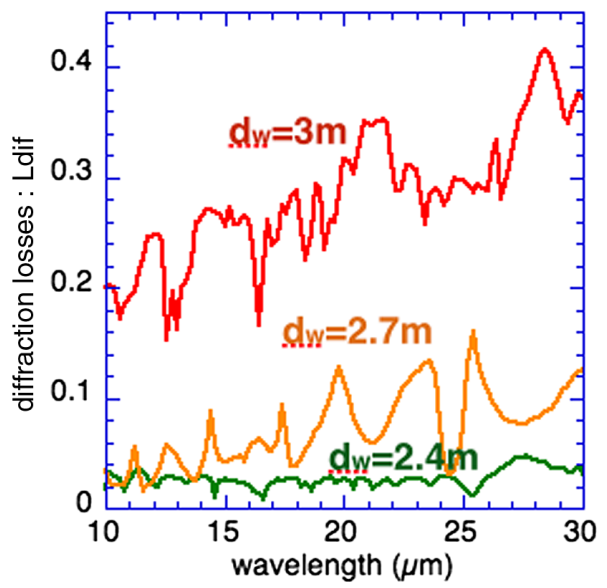

FIG. 15. Diffraction losses $L_{\text {dif }}$ versus wavelength at various waveleguide length $d_{w}$.

This phenomenon is well explained when observing the transverse profile of the laser amplitude $A(x, y)$ calculated at the waveguide exit (point A in Fig. 9). Figure 16 displays these profiles, at $\lambda=10 \mu \mathrm{m}$ and $20 \mu \mathrm{m}$, for different values

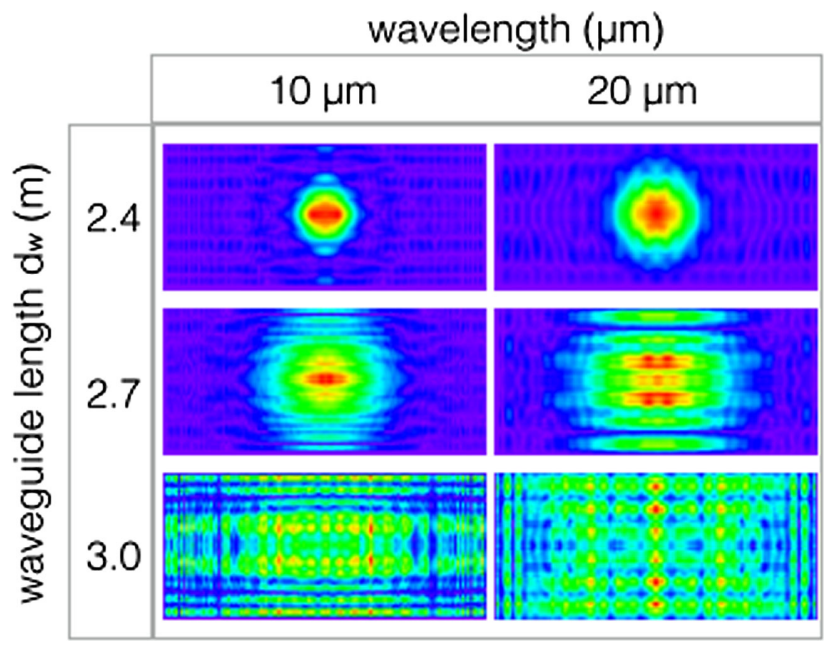

FIG. 16. Amplitude profile of the laser, at the waveguide output (point $\mathrm{A}$ in Fig. 9), for various wavelength $\lambda$ and waveguide length $d_{w}$.

of the waveguide length near the threshold value $d_{w} \cong 2.7 \mathrm{~m}$. At small length $d_{w}<2.7 \mathrm{~m}$ the spot remains close to a round shape, but for higher values it exhibits a complex structure, and it fills the whole transverse size of the waveguide for $d_{w} \geq 3 \mathrm{~m}$.

This complex structure gives a large optical diffraction in the free space areas close to the mirrors. Figure 17 shows the laser profile for $\lambda=20 \mu \mathrm{m}$ and $d_{w}=3 \mathrm{~m}$, on the upstream mirror (point B in Fig. 9) and on the upstream entrance of the waveguide (point $\mathrm{C}$ in Fig. 9).

The dimensions of the mirror and of the waveguide are represented by a white line shape. The amount of lost energy, outside of these limits, corresponds to losses of $14 \%$ and $16 \%$ respectively. Therefore, the diffraction is responsible for the large amount of optical losses upon the mirrors and at the waveguide entrances. This effect is not obvious to predict intuitively, because, in principle, the waveguide allows us to drive the wave without energy losses, and its length should not be an inconvenience. Nevertheless, it seems to be a crucial parameter when it is placed inside an optical cavity. In this configuration, the
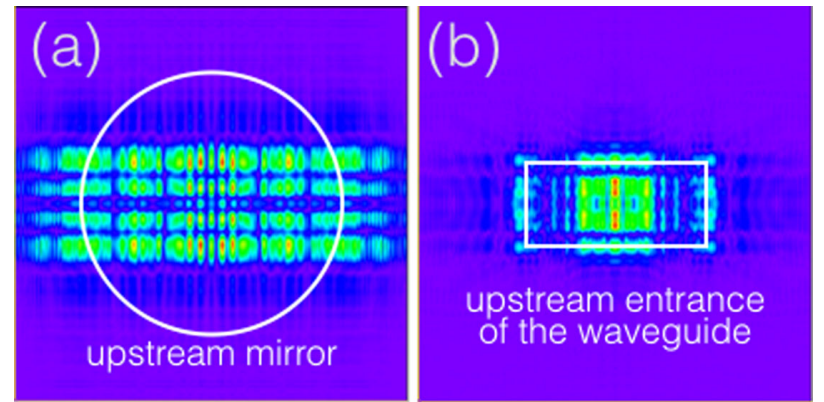

FIG. 17. Amplitude profile of the laser, for $\lambda=20 \mu \mathrm{m}$ and $d_{w}=3 \mathrm{~m}$, (a) on upstream mirror (point B in Fig. 9), and (b) on waveguide entrance (point $\mathrm{C}$ in Fig. 9). 


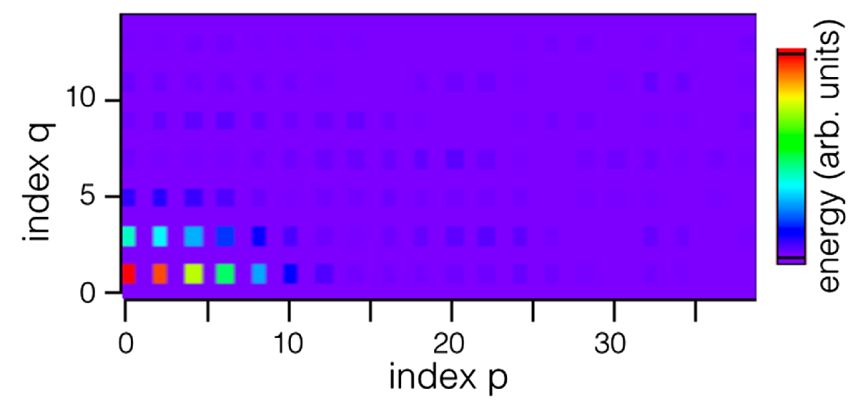

FIG. 18. Energy of the eigenmodes $\mathrm{TEM}_{p q}$ for CLIO at $\lambda=10 \mu \mathrm{m}$ and $d_{w}=2 \mathrm{~m}$.

problem comes from the diffraction outside of the waveguide. As a consequence, in order to overcome this problem of diffraction, a full length waveguide, without free space, seems to a good solution. Nevertheless, we will see below that problems of mode scattering are still present when using such a full waveguide cavity.

\section{B. Eigenmode distribution in the waveguide section}

This discussion does not involve the notion of eigenmodes in the waveguide section, but it is interesting to see the distribution of eigenmode of CLIO, and compare to the pattern displayed in Fig. 8 corresponding to the configuration of FLARE. Figure 18 gives the amplitude of TEM $p q$, for CLIO at $\lambda=10 \mu \mathrm{m}$ and nominal waveguide length $d_{w}=2 \mathrm{~m}$, as a function of indexes $(p, q)$. The displayed range is limited to the modes $p \leq 39$ and $q \leq 15$, which contain $90 \%$ of the total energy (the whole set contains $128 \times 128$ modes). Unlike the case of FLARE, the distribution is rather homogeneous between $p$ and $q$. Indeed, the waveguide aperture of CLIO $(35 \times 16 \mathrm{~mm})$ is not as flat as for FLARE $(200 \times 1 \mathrm{~cm})$, which the mode distribution extension is $p \leq 95$ and $q=1$.

\section{NUMERICAL SIMULATION USING A FULL CAVITY WAVEGUIDE}

With the previous configuration, the diffraction losses occur in the free space areas, on the mirrors and at the ends of the waveguide. Using a full waveguide in the cavity allows us to avoid these diffraction losses. Figure 19 displays a layout of such a configuration, using plane mirrors (because no free space), and still using a $2 \mathrm{~mm}$ hole

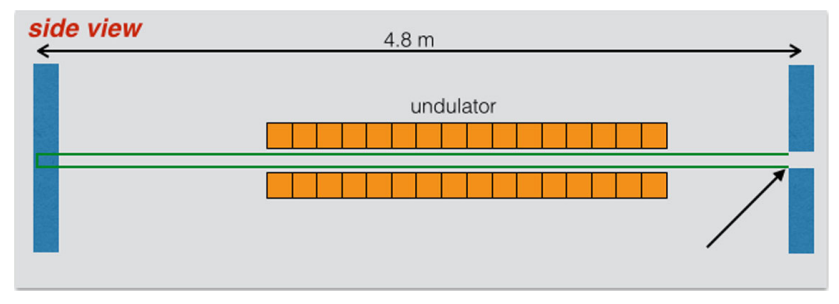

FIG. 19. Layout of the FEL with a full waveguide configuration. (a)

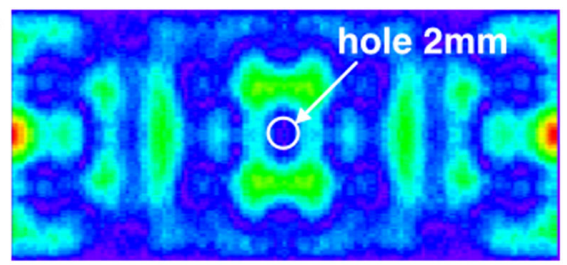

(b)

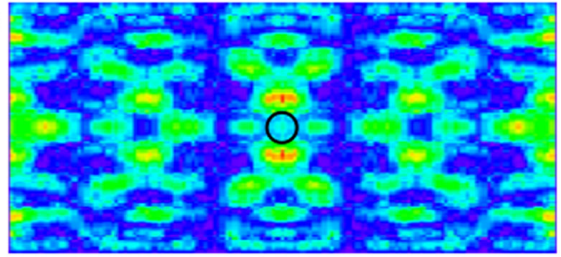

(c)

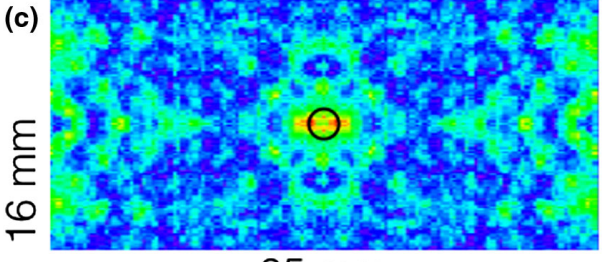

$35 \mathrm{~mm}$

FIG. 20. Amplitude profile of the laser on output mirror, for (a) $\lambda=19.9 \mu \mathrm{m}$, (b) $\lambda=20 \mu \mathrm{m}$, (c) $\lambda=20.1 \mu \mathrm{m}$.

coupling on the output mirror. The waveguide length is $d_{w}=4.8 \mathrm{~m}$. Note that, a perfect full waveguide is not realistic in a FEL design, because it is necessary to use open walls on the side of the waveguide in order to allow electron beam passing.

In this case, as shown in Fig. 20, the laser profile exhibits a complex structure. These profiles have been calculated on the output mirror, at 3 different wavelengths close to $\lambda \cong 20 \mu \mathrm{m}$. A small wavelength step of $0.1 \mu \mathrm{m}$ allows the profile to change completely. This behavior is observed at any wavelength within the tuning range of the laser. The hole extraction is placed in the center of the waveguide transverse area, and it takes the energy from the center of the profile distribution. Therefore, the extraction rate $T_{x}$, and the output power, also follow a quite random behavior according to the wavelength.

Table III gives the losses $L_{\mathrm{os}}$ and extraction rate $T_{x}$ obtained with the 3 profiles displayed in Fig. 20: the extraction rate is jumping by a factor 40 (note that the total losses are equal to the sum of $T_{x}$ with $4 \%$ of absorption on mirrors). The output power versus wavelength is shown in Fig. 21, and as expected, it exhibits a strong spiky behavior.

TABLE III. Total losses $L_{\mathrm{os}}$ and extraction rate $T_{x}$ versus wavelength corresponding to the profiles of Fig. 20.

\begin{tabular}{lccc}
\hline \hline & a) $19.9 \mu \mathrm{m}$ & b) $20 \mu \mathrm{m}$ & c) $20.1 \mu \mathrm{m}$ \\
\hline Total losses Los & $4.1 \%$ & $4.5 \%$ & $7.4 \%$ \\
Extraction rate Tx & $0,08 \%$ & $0.5 \%$ & $3.3 \%$ \\
\hline \hline
\end{tabular}




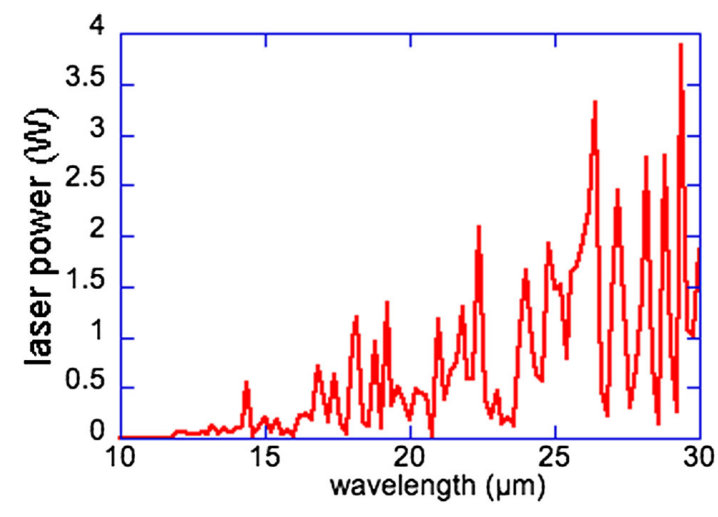

FIG. 21. Laser power versus wavelength.

In fact, the plane mirrors, when used in a full waveguide cavity, do not alter the laser propagation in the cavity (apart from the hole coupling and the gain), and such configuration is equivalent to a waveguide of infinite length. As a consequence, if the cavity length (and the waveguide) is shorter than $2.5 \mathrm{~m}$, which corresponds to the threshold value $d_{w}$ obtained in Sec. III, the laser profile still exhibits a complex structure, with s spiky output laser power. This has been checked by the simulation for $d_{w}<2 \mathrm{~m}$. We also have studied the influence of the radius of curvature of the cavity mirrors, and the result is the same: when replacing the plane mirrors by concave mirrors, with a radius of curvature $R_{c}=3 \mathrm{~m}$ (like in the CLIO usual cavity), the output laser remains spiky and the laser power dependence on wavelength also. As a summary, in the usual CLIO configuration, the free space areas, at both end of the waveguide, are creating diffraction losses, but they are also smoothing the laser mode in the cavity, and this is important to avoid the spiky phenomenon which is produced in the waveguide section.

\section{A. Without hole coupling}

Two elements are still able to modify the laser profile in the cavity: the hole coupling and the gain medium. The extraction hole makes a scattering of light inside the cavity, and it has an influence upon the transverse profile of the laser. In order to check this, we use the above configuration (full waveguide and plane mirrors) without extraction hole: the laser extraction is achieved using a transmission mirror of $\left(1-R_{M}\right)=0.02$. Note that broadband transmission mirrors are not realistic for infrared range, because no wideband dichroic mirror exist in infrared, and most part of substrates contain absorption bands. The result of simulation, close to $\lambda \cong 20 \mu \mathrm{m}$, is displayed in Fig. 22: even without hole coupling, the profiles exhibit a complex structure.

With this last cavity configuration, both the total losses $L_{\mathrm{os}}=4 \%$ and the extraction rate $T_{x}=2 \%$ are independent of wavelength. They are only dependent on the output mirror reflexion $R_{M}=0.98$, which is kept constant. (a)

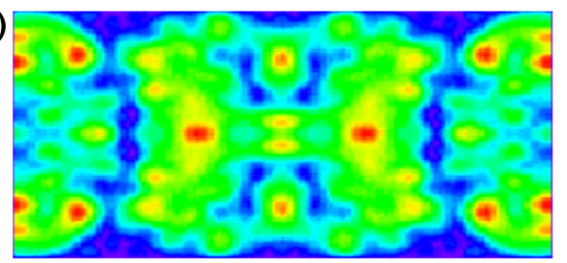

(b)
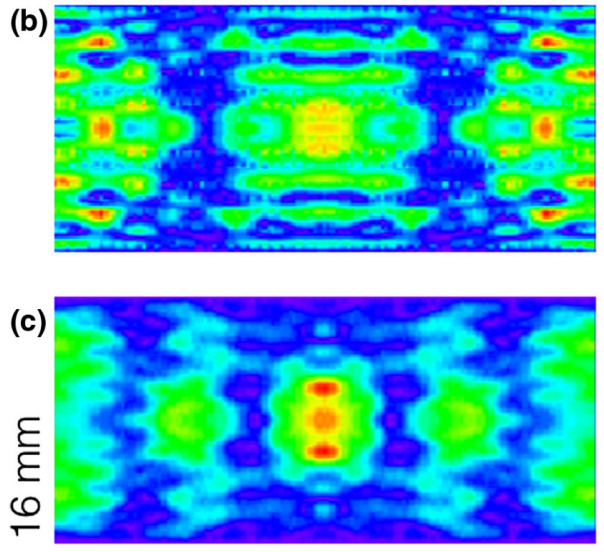

$35 \mathrm{~mm}$

FIG. 22. Amplitude profile of the laser on output mirror without extraction hole, for (a) $\lambda=19.9 \mu \mathrm{m}$, (b) $\lambda=20 \mu \mathrm{m}$, (c) $\lambda=20.1 \mu \mathrm{m}$.

Therefore, it would be reasonable to expect a constant output laser power, or at least a rather smooth variation according to wavelength (due to intrinsic FEL gain $G^{*}$ variations). Nevertheless, as shown in Fig. 23, the laser power still shows a spiky behavior; less strong than for the previous case with a hole coupling, but still visible.

This is due to the small transverse size of the gain medium, i.e., the electron beam, $\sigma_{e}=0.8 \times 0.4 \mathrm{~mm}$ in our case. Indeed, the gain only occurs in a small area, in the center of the transverse distribution in the waveguide (small area as compared to the whole area of the waveguide $35 \times 16 \mathrm{~mm}$ ). The required gain $g_{o}$ is then strongly

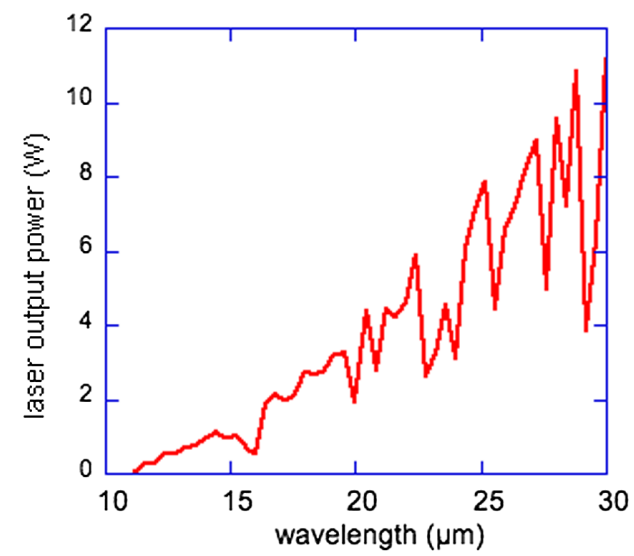

FIG. 23. Laser power versus wavelength, without extraction hole (transmission mirror). 
dependent on the "filling factor" $F_{f}=\Sigma_{G} / \Sigma_{W}$, which represents the ratio between (1) the area $\Sigma_{G}$ of the gain distribution, and (2) the area $\Sigma_{W}$ of the wave (which fits the waveguide transverse size as shown in the above profiles). Indeed, when the central part of the profile exhibits a small amplitude, the gain coefficient must be strong enough to compensate the cavity losses. The gain coefficient is then very sensitive to the distribution of the laser profile. Therefore, several consequences are induced by this effect: (1) the laser mode is forced to have a nonzero amplitude in the center of the profile, (2) this requirement is responsible for the complex pattern of the laser transverse profile, and (3) the relevant variations of the gain coefficient are creating laser power spiky variations according to the wavelength. This is well observed in Fig. 23.

\section{B. Uniform transverse distribution of the gain}

In order to check this effect, the electron beam transverse size has been extended to large values $\sigma_{e}=10 \times 10 \mathrm{~mm}$, still keeping transmission mirror for output (no hole coupling). Such beam size is not realistic in practice, because one part of the electron beam would hit the waveguide walls. In this case, the transverse distribution of gain is quite uniform, it does not disturb the laser mode, and the gain coefficient is not affected by the distribution of the laser profile. Also, the configuration of the FEL is now equivalent to an infinite waveguide, as no element may affect the laser mode (no focusing, distortion nor clipping of the mode). Figure 24 displays the profile obtained at

(a)

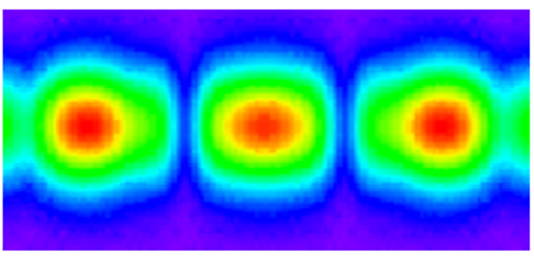

(b)

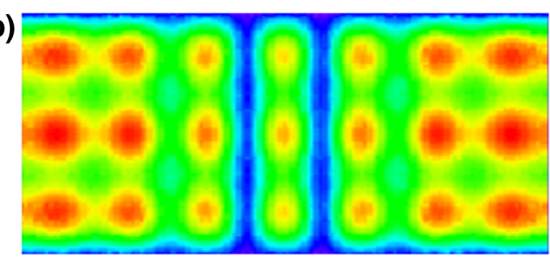

(c)

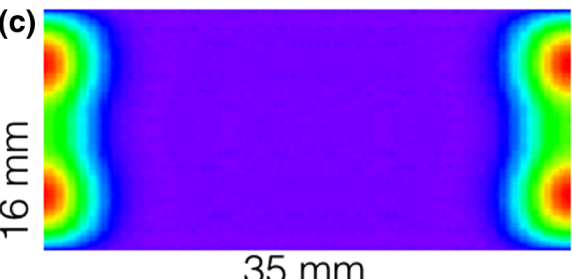

$35 \mathrm{~mm}$

FIG. 24. Laser profiles on output mirror without hole, and with broad gain profile, at $\lambda=20 \mu \mathrm{m}$, for consecutive round trips $N_{c}$ in the cavity: $N_{c}=17$ (a), 18 (b) and 19 (c). $\lambda=20 \mu \mathrm{m}$, after various consecutive round trips $N_{c}$ of the laser wave in the cavity.

No convergence occurs within this configuration, because the wave propagates freely in the infinite waveguide, and the interferences of eigenmodes are producing a permanent variation of the laser profile all along the waveguide propagation. We have checked that the profile jumps, like in Fig. 24, at each round trip up to $N_{c}>300$ and without stabilization. The "infinite" width of gain medium allows a large number of different laser profiles: (B) a broad profile within the full waveguide area, or (C) on energy distribution on edges with zero amplitude in the center.

Note that such permanent variation of the laser profile along the propagation does not occur with the other configurations, where the laser profile is forced to fit the small gain transverse area, and which the convergence leads to a stable profile at saturation regime. In this case, during the convergence process, the laser mode starts from the "jumping regime" described above (Fig. 24), and goes toward the stable pattern of Fig. 22(b). Figure 25(a) displays some steps of such convergence process at $\lambda=20 \mu \mathrm{m}$, using a small cross section of gain. A comparison is shown in Fig. 25(b) using a large cross section, and which does not converge.

\section{Uniform gain and hole coupling}

As a first summary of this analysis, two parameters are responsible for the spiky behavior of the laser mode when using a waveguide in the cavity: (1) small transverse size of the gain medium, and (2) hole coupling. However, it is necessary to study the last combination: uniform gain and hole coupling. This configuration, using a hole coupling of $2 \mathrm{~mm}$, gives a profile displayed in Fig. 26 for $\lambda=20 \mu \mathrm{m}$, (a) $\sigma=0.8 \times 0.4 \mathrm{~mm}$
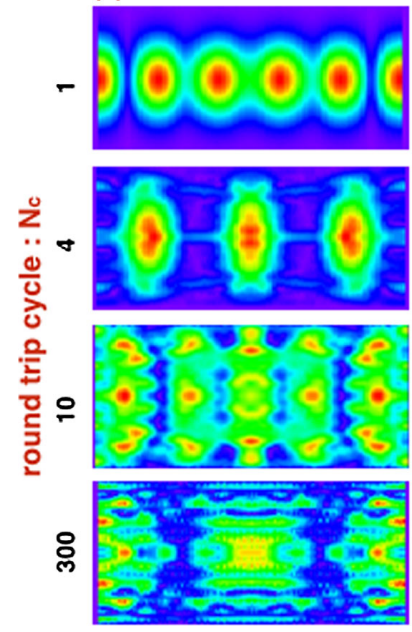

(b) $\sigma=10 \times 10 \mathrm{~mm}$
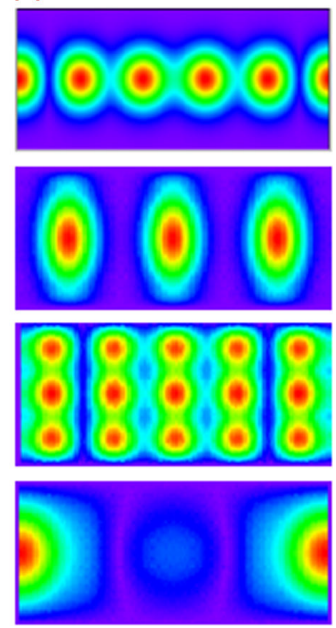

FIG. 25. Profile convergence vs cavity round trip $N_{c}$, at $\lambda=20 \mu \mathrm{m}$, (a) using a small cross section of gain, (b) and a large one. 


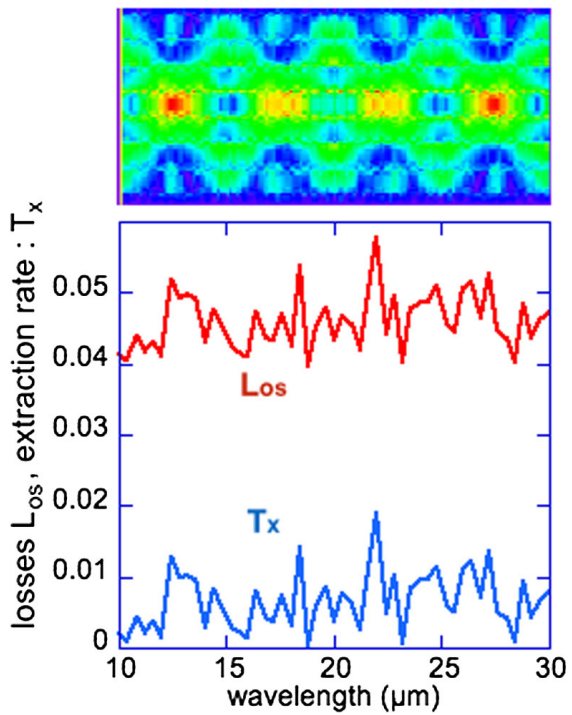

FIG. 26. Profile at $\lambda=20 \mu \mathrm{m}$, using a uniform gain and a $2 \mathrm{~mm}$ hole coupling, and curve of losses and extraction rate vs wavelength.

and the extraction rate $T_{x}$ and losses $L_{\mathrm{os}}$ versus wavelength. They also show a spiky behavior.

Using a vertical extraction slit, instead of a hole, seems more adapted to a horizontal waveguide, especially for a rather flat waveguide as for the FLARE FEL which uses a slit of $2.5 \mathrm{~mm}$ width and a waveguide of $200 \times 10 \mathrm{~mm}$. A simulation has been done within the above conditions but using a slit of $2.5 \mathrm{~mm}$ width. The result is displayed in Fig. 27. The pattern shows vertical lines with a quite random distribution, and the extraction rate $T_{x}$ is spiky.

These last simulations, using a uniform gain or a hole coupling, show a spiky behavior. The small transverse size

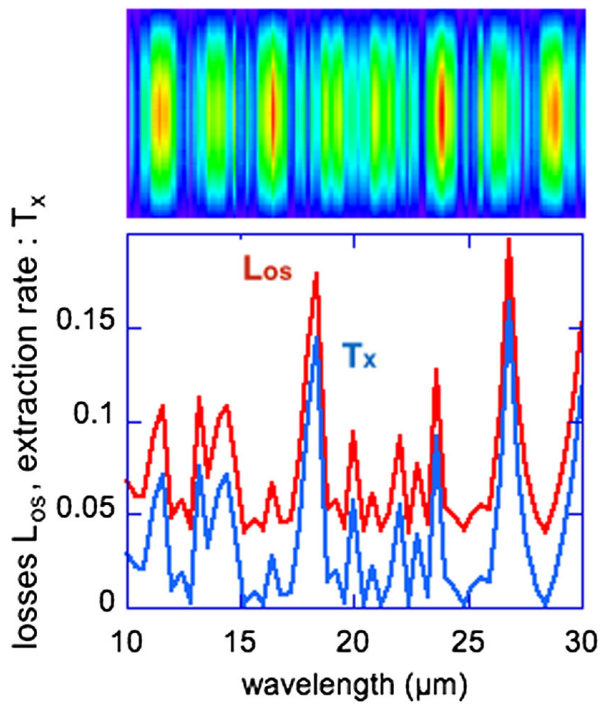

FIG. 27. Profile at $\lambda=20 \mu \mathrm{m}$, with a uniform gain and a slit of $2.5 \mathrm{~mm}$ width, and curve of losses and extraction rate vs wavelength. of gain (1) and the hole coupling (2) can be considered as equivalent perturbations acting upon the wave front. Indeed, (1) corresponds to a local amplification of the wave (within the gain transverse size), whereas (2) corresponds to a local attenuation (within the hole transverse size). Therefore, the influences of (1) and (2) upon the wave front are equivalent, though giving opposite effects. In order to cancel the spiky behavior, no one of these conditions shall occur: the cavity must have uniform gain and no hole coupling. The solution of cylindrical mirrors, as designed for FLARE, allows us to approach the condition of a uniform gain, since the wave is focused in the gain medium. However, it seems more difficult to avoid the hole coupling extraction process, because of properties of optical materials in infrared range (absorptions and variations of refractive index).

\section{FULL CAVITY WAVEGUIDE AND CYLINDRICAL MIRRORS, WITHOUT EXTRACTION SLIT}

Using cylindrical mirrors [12] for the cavity allows us to focus the laser, in horizontal, upon the electron beam in the undulator section. It corresponds to the configuration of FLARE, described in Sec. II, which the parameters are used in the simulations below, except that no extraction slit is used here: the extraction is achieved by transmission through the output mirror. Figure 28 displays the laser profiles in the cavity at various wavelength: the waveguiding effect clearly occurs, along vertical axis exclusively. Figure 28(a) shows the laser profile in the plane of gain, in the middle of undulator section. The RMS transverse size of gain distribution $\sigma_{X Y}=5 \times 4 \mathrm{~mm}$ is represented to scale by a black dot. The profile on output mirror is displayed in Fig. 28(b). The distribution exhibits a complex structure in the vertical axis, due to wave-guiding, and a smooth one in the horizontal axis. This can be compared to Fig. 6 using an output slit, which makes a diffraction of the wave inside the cavity and leads to spiky features. The laser focusing in horizontal has various consequences: (1) the "filling factor" $F_{f}$ of the gain (see in Sec. IV) is increased because the gain distribution fits

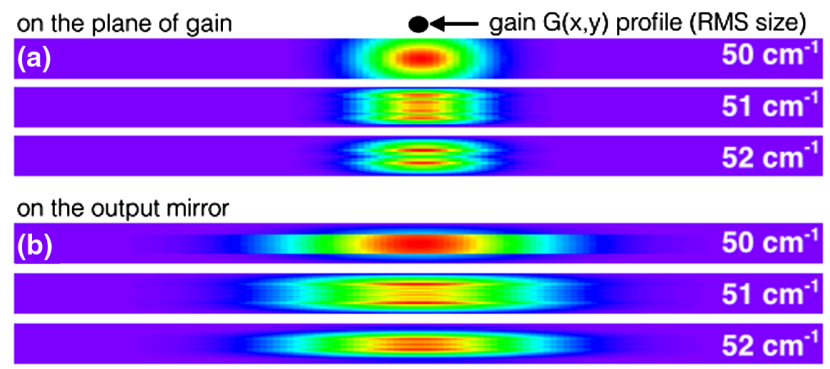

FIG. 28. Laser profile amplitude in the waveguide $(200 \times 1 \mathrm{~cm})$ : (a) on the plane of gain and (b) on the output mirror, at various wavelength. 


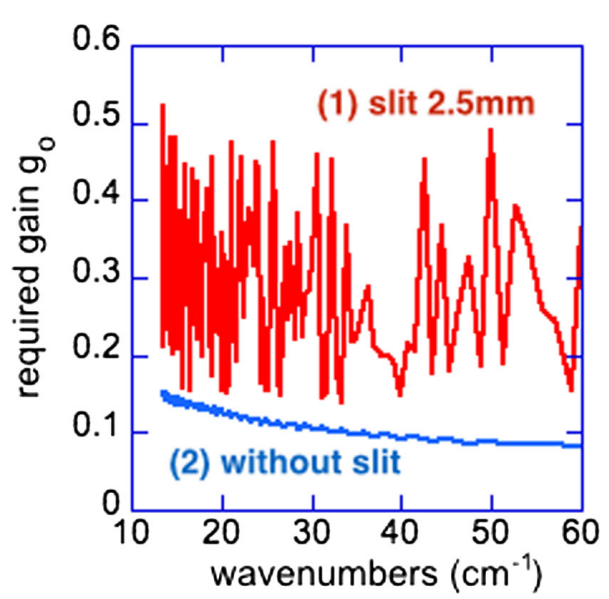

FIG. 29. Required gain coefficient $g_{o}$ vs wavelength, (1) with extraction slit, and (2) with a transmission mirror (without slit).

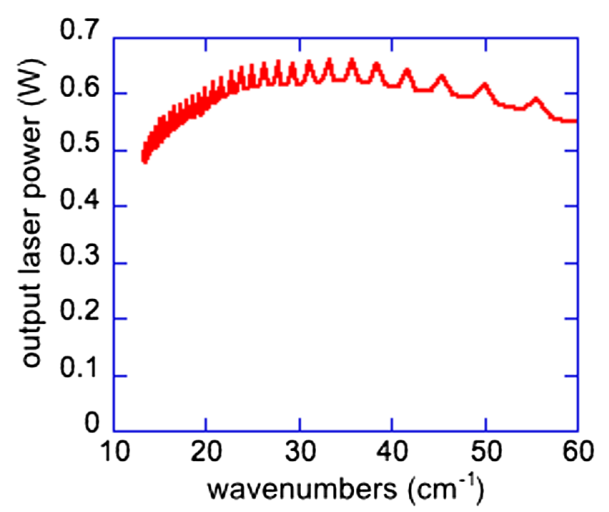

FIG. 30. Simulation of the output laser power of FLARE, without extraction slit (extraction by mirror transmission).

better to the laser profile, (2) the amplification of the wave is more uniform in transverse plane, (3) and it forces the wave to have a maximum of amplitude on axis, (4) the required gain coefficient $g_{o}$ is independent on the distribution of the laser profile, and its required value is less important: see Fig. 29. As a consequence, focusing has a crucial and beneficial effect on the laser mode, and the output laser power has a soft dependence to wavelength: see Fig. 30.

\section{CONCLUSION}

These simulations and comments are showing that, when a full waveguide is used inside the optical cavity, the transverse distribution of laser exhibits a complex pattern, and the output power shows spiky variations according to wavelength. This phenomenon is due principally to: (1) the hole coupling (or slit) used for laser extraction, or (2) the small extension of the transverse distribution of the FEL gain. More generally, the fundamentals of this phenomenon are intrinsic to overmoded waveguides, which are driving the wave with a nonstable transverse profile.

The hole coupling forces the laser profile to have a nonzero amplitude on axis, in order to improve the extraction, but it creates diffraction in the cavity. This makes a complex structure on the laser transverse profile, which gives random extraction rate for output coupling, random losses and spiky power variations according to the wavelength.

The small cross section of the gain distribution also forces the laser to have a nonzero amplitude on electron beam axis. Though its influence is less than hole coupling (because no diffraction effect) it has the same kind of influence than the hole: it makes a local amplification of the wave in the cavity, and also gives a spiky features. Using cylindrical mirrors improves the behavior, because it makes a focusing of the wave into the gain cross section, which allows us to approach the condition of a uniform gain.

Using a transmission mirror, or a Brewster plate, for laser extraction (i.e., without hole extraction), and cylindrical mirrors for the cavity, allows us to get a smoothed laser profile, and smooth variations of laser power according to the wavelength. However this solution requires an output mirror, or an extraction plate, with nonabsorbing optical material in the large wavelength range of the FEL. And if using a Brewster plate, the index variations will change slightly the tuning length of the cavity.

No such effect happens with an opened cavity, i.e., without waveguide, but in this case the optical diffraction in undulator vacuum chamber makes a limitation at large wavelength range. A partial waveguide cavity, using a waveguide only in the undulator section and free space areas close to the cavity mirrors, represents a compromise between these two situations. The optical diffraction in free spaces makes a smoothing of the laser profile, and allows a blending of eigenmodes in the waveguide. However, we have shown that the length of the waveguide must be limited, for example less than $2.7 \mathrm{~m}$ in the case of CLIO, otherwise the laser profile exhibits a complex pattern in the waveguide which creates large losses by diffraction in the free space areas. This complex pattern is intrinsic to overmoded waveguides, as discussed above.

\section{ACKNOWLEDGMENTS}

We are thankful to Lex van der Meer, and more generally to the FELIX and FLARE free-electron laser group, for their contribution by sending us experimental data of the FEL.

[1] D. A. G. Deacon, L. R. Elias, J. M. J. Madey, G. J. Ramian, H. A. Schwettman, and T. I. Smith, First Operation of a Free-Electron Laser, Phys. Rev. Lett. 38, 892 (1977). 
[2] W. B. Colson, J. Blau, K. Cohn, J. Jimenez, and R. Pifer, in Free-electron lasers conference, Liverpool, U.K., 2009 (Joint Accelerator Conferences, CERN, Geneva, Switzerland, http://cern.ch/AccelConf/FEL2009/papers/wepc43 .pdf.

[3] Li Yi Lin and A. F. G. van der Meer, Design of a shortpulse, far-infrared free electron laser with a highly overmoded waveguide, Rev. Sci. Instrum. 68, 4342 (1997).

[4] R. Prazeres, F. Glotin, and J. M. Ortega, New results of the CLIO infrared FEL, Nucl. Instrum. Methods Phys. Res., Sect. A 528, 83 (2004).

[5] P. Michel et al., in Proceedings FEL 2006-BESSY, Berlin, Germany (Joint Accelerator Conferences, CERN, Geneva, Switzerland, p. 488 http://cern.ch/AccelConf/f06/ PAPERS/TUCAU02.PDF.

[6] D. Arslanov et al. in Proceedings of the 36th International Free Electron Laser Conference, Basel, Switzerland, 2014 (Joint Accelerator Conferences, CERN, Geneva, Switzerland, http://accelconf.web.cern.ch/AccelConf/FEL2014/ posters/tup065_poster.pdf.

[7] R. Prazeres, F. Glotin, and J.-M. Ortega, Analysis of periodic spectral gaps observed in the tuning range of free-electron lasers with a partial waveguide, Phys. Rev. Accel. Beams 12, 010701 (2009).

[8] R. Prazeres and M. Billardon, Numerical calculation of transverse optical modes for characterisation of an optical cavity, Nucl. Instrum. Methods Phys. Res., Sect. A 318, 889 (1992).

[9] R. Prazeres, Numerical method for full calculation of the electromagnetic field in a rectangular waveguide within overmoded configuration, using the fast Fourier transform, Eur. Phys. J. Appl. Phys. 68, 20501 (2014).

[10] R. Prazeres, F. Glotin, and J. M. Ortega, Measurement, and calculation of the Electron efficiency on the CLIO FreeElectron Laser, Eur. Phys. J. Appl. Phys. 29, 223 (2005).

[11] G. Dattoli, Logistic function, and evolution of freeelectron-laser oscillators, J. Appl. Phys. 84, 2393 (1998).

[12] L. R. Elias and J.C. Gallardo, Cylindrical GaussianHermite modes in rectangular wave guide resonators, Appl. Phys. B 31, 229 (1983).

[13] J. W. J. Verschuur, G. J. Ernst, B. M. van Oerle, and D. Bisero, The effect of transverse modes in a wave-guide resonator on the resonance condition of a Compton, Opt. Commun. 133, 229 (1997). 